\title{
COLECTA DE ANTOCÓRIDOS Y DETERMINACIÓN DE SU CAPACIDAD DEPREDADORA SOBRE ADULTOS DE Frankliniella occidentalis (PERGANDE) (THYSANOPTERA, THRIPIDAE)
}

Fecha de recepción: 13 de enero de 2015 • Fecha de Evaluación: 25 de noviembre de 2015 • Fecha de aceptación: 7 de marzo de 2016

\section{COLLECTION OF ANTHOCORIDS AND DETERMINATION OF THEIR PREDATORY CAPACITY ON ADULTS OF Frankliniella occidentalis (PERGANDE) (THYSANOPTERA, THRIPIDAE)}

Jhon Alexander Avellaneda Nieto', Marco Antonio Díaz Tapias'1, Daniel Augusto Acosta Leal', Daniel Rodríguez Caicedo ${ }^{1 *}$, Fernando Cantor Rincón ${ }^{1}$

\section{RESUMEN}

El trips Frankliniella occidentalis, actualmente es la plaga cuarentenaria más importante en cultivos de flores de la sabana de Bogotá, en Colombia es la especie que registra más interceptaciones, lo que causa grandes pérdidas económicas, por esto, es importante encontrar estrategias de manejo de esta plaga, como lo es control biológico. Los antocóridos del genero Orius son reconocidos agentes de control biológico los cuales depredan trips F. occidentalis. Este estudio tuvo como objetivo, colectar e identificar antocóridos presentes naturalmente en seis fincas floricultoras de la sabana de Bogotá y determinar su capacidad depredadora sobre adultos de F. occidentalis. Se realizaron muestreos en seis fincas floricultoras ubicadas en la sabana de Bogotá. En cada finca, se realizó el muestreo en la flora circundante a los invernaderos. Simultáneamente se evaluaron cuatro métodos de muestreo: sacudida del follaje, jameo, golpeteo y caída. Los individuos de las especies colectadas fueron identificados taxonómicamente y se estableció un pie de cría de cada una, para realizar el ensayo de capacidad de depredación. De 394 individuos colectados el 75\% correspondió a 0 . insidiosus, 24\% a O. pumilio y $1 \%$ a una especie de antocórido que no pertenecía al género Orius. El método de colecta más exitoso fue el de golpeteo, con $67 \%$ de las capturas, seguido por el de jameo con 33\%. No se capturaron individuos con los otros métodos. La especie O. insidiosus consumió en promedio 23 individuos adultos de F. occidentalis en 24 horas, siendo significativamente mayor al consumo encontrado para las otras dos especies.

Palabras Clave: Métodos de muestreo, Cercas vivas, Orius insidiosus, Orius pumilio.

1 Laboratorio de Control Biológico, Facultad de Ciencias Básicas y Aplicadas, Universidad Militar Nueva Granada, Cajicá, Colombia, Km 2 Vía Cajicá-Zipaquirá

*Autor Corresponsal. E-mail: control.biologico@unimilitar.edu.co; Tel: (57) 6500000 Ext: 3269 


\begin{abstract}
Frankliniella occidentalis, currently is a major flowers crops quarantine pest in Bogotá plateau. In Colombia is the most intercepted pest, causing substantial economic losses. It is important to find management strategies of this pest, as biological control. Anthocorids of genus Orius are recognized biological control agents that depredate thrips F. occidentalis. The goal of this study was to collect and identify Anthocorids that occur naturally in Bogotá plateau flowers crops and determinate their predation capacity on F. occidentalis adults. In six flowers farms, samples were taken from several plants around greenhouses. Simultaneously were evaluated four different sampling methods: beating foliage, "golpeteo", sweep-net and pitfall traps. The insects collected were taxonomically identified and a stock colony was started. A predation capacity test was conducted. Of 394 individuals collected the $75 \%$ was identified as 0 . insidiosus, $24 \%$ as 0 . pumilio, and $1 \%$ of individuals collected as not beloging to Orius genus. The sampling method more successful was the "golpeteo" wasthe "golpeteo" with 67\% of the collections, followed by the sweep-net method with the 33\%. Individuals were not collected using the other methods. Significant differences in the mean consumption of $F$. occidentalis adults were observed for $O$. insidiosus (23 individuals $/ 24 \mathrm{~h}$ ) respect to the other species.
\end{abstract}

Key words: Sampling methods, hedges, Orius insidiosus, Orius pumilio.

\title{
INTRODUCCIÓN
}

Los trips (Insecta:Tysanoptera) comprenden un numero amplio de especies fitófagas, cosmopolitas y comúnmente, son plaga de un gran número de especies vegetales (Hill, 2008). Hospedan tanto especies cultivables, como especies silvestres (Tommasini y Maini, 1995). En los cultivos ornamentales, frutales y hortícolas que estas plagas afectan, en muchos casos, ocasionan daños por encima del umbral de daño económico (Childers y Nakahara, 2006).

Lo anterior, se constituye en un gran problema para los agricultores en muchos países del mundo, especialmente, en los países tropicales (Lewis, 1997; Johansen y Mojica, 2007). Los agricultores colombianos, no son ajenos a este problema, en el país, se han reportado diferentes estudios sobre la presencia de especies de trips, los cuales afectan diferentes cultivos, especialmente en la sabana de Bogotá (Zapata et al. 1994; Vergara, 1998). Un número amplio de especies de trips, se encuentran comúnmente en la sabana de Bogotá, Calixto (2005) reporto 79 especies de trips en esta región del país y muchas de estas, asociadas a cultivos de flores como plaga.

Frankliniella occidentalis es una especie de trips plaga, la cual es cosmopolita, cuarentenaria y constituye uno de los principales problemas en cultivos comerciales, en la sabana de Bogotá es una de las plagas con mayor importancia en las fincas floricultoras, causa daños directos sobre las plantas, provocados al succionar el contenido celular de hojas, flores y frutos (Demirozer et al. 2012), lo que ocasiona una serie de manchas color gris-plateado, las cuales posteriormente se necrosarán (Hunter y Ullman, 1989), también causa daños debido a la secreción de sustancias fitotoxicas, al alimentarse y al insertar el aparato ovopositor en los tejidos de la 
planta, esto conduce a la distorsión o deformación de los órganos de la planta (Parker et al. 1995; Childers y Achor, 1995; Bosco et al. 2007).

Todos los daños mencionados anteriormente, son considerados daños cosméticos, los cuales impiden la comercialización de las flores. Gran parte de la producción de flores en Colombia es destinada para la exportación, de la cual el $85 \%$ se concentra en Cundinamarca y especialmente en la en la sabana de Bogotá (Tenjo et al. 2006). Para la exportación de los cargamentos de flores se realizan inspecciones fitosanitarias antes de entrar a los países de destino, con el propósito de interceptar plagas, en caso de interceptar plagas cuarentenarias como los trips, los cargamentos son fumigados en algunos casos, y en otros casos, devueltos o incinerados, lo que representa grandes pérdidas económicas para los floricultores, según el Instituto Colombiano Agropecuario (ICA), la mayor proporción de las interceptaciones realizadas por las entidades aduaneras de los países donde se exportan estos productos, son ocasionadas por trips; en el año 2013 se reportaron aproximadamente 700 interceptaciones, esto genero grandes pedidas al sector floricultor (Mendoza, 2014).

El método más utilizado para el control de trips, es el químico, sin embargo, a causa de los hábitos crípticos de la plaga, no se ha alcanzado la eficiencia esperada (Castresana et al. 2008). Además, este método de control presenta desventajas como lo es la selección de poblaciones con genes resistentes (Bielza, 2008), lo cual produce un aumento, tanto de la frecuencia de aplicación de los productos químicos, como de las dosis aplicadas, que termina en un incremento en los costos de producción del cultivo y un alto impacto al medio ambiente y a la salud humana (Bajonero et al. 2008). Por este motivo es necesario y de gran importancia atacar este problema por medio de alternativas de manejo menos perjudiciales para el medio ambiente y el agricultor, como es el caso del control biológico con especies nativas o que incidan de manera natural en los agroecosistemas.

Dentro de los agentes de control biológico que se ha demostrado que tienen efecto sobre la regulación de poblaciones de trips, se encuentran los antocóridos (Hemiptera: Anthocoridae), especialmente del genero Orius. Desde hace algunos años en Europa, se comercializan como bioinsumo antocóridos, para la regulación de trips en diferentes cultivos (Van Lenteren et al. 1997; Shipp y Ramakers, 2004). Este trabajo tuvo como objetivo colectar e identificar antocóridos presentes naturalmente en fincas floricultoras de la sabana de bogotá y determinar su capacidad de depredación sobre trips F. occidentalis adultos, bajo condiciones de laboratorio.

\section{MATERIALES Y MÉTODOS}

Colecta e identificación taxonómica de antocóridos

\section{Área de estudio}

Con el propósito de colectar los individuos del genero Orius, se seleccionaron seis fincas productoras y comercializadoras de flores, las cuales estaban distribuidas en la región sabana centro y sabana occidente en el departamento de Cundinamarca, las fincas estaban ubicadas en cinco municipios, en Cajicá, Chía, Tocancipá y Facatativá se seleccionó una finca y en el municipio de Sopo dos fincas, en las siguientes coordenadas geográficas: Cajicá (4 $57^{\prime}$

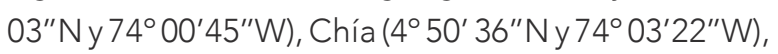

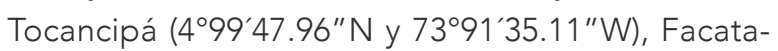
tivá ( $4^{\circ} 47^{\prime} 33^{\prime \prime} \mathrm{N}$ y $\left.74^{\circ} 21^{\prime} 29^{\prime \prime} \mathrm{W}\right)$, y dos fincas en el

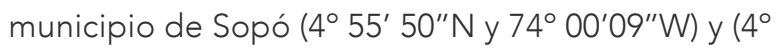
$55^{\prime} 18^{\prime \prime} \mathrm{N}$ y $\left.73^{\circ} 58^{\prime} 12^{\prime \prime} \mathrm{W}\right)$.

En cada finca se seleccionaron puntos de muestreo (Tabla 1), los cuales estaban ubicados en los alrededores de los invernaderos. El criterio de selección de los puntos de muestreo, se basó en la 
Tabla 1. Número de puntos muestreados en cada finca, discriminado por método de muestreo.

\begin{tabular}{cccccccc}
\hline Método/finca & Finca 1 & Finca 2 & Finca 3 & Finca 4 & Finca 5 & Finca 6 & Total \\
\hline $\begin{array}{c}\text { Sacudida } \\
\text { del follaje }\end{array}$ & 6 & 5 & 5 & 3 & 1 & 1 & 21 \\
\hline Trampa de caída & 5 & 5 & 5 & 5 & 5 & 5 & 30 \\
\hline Jameo & 21 & 13 & 15 & 23 & 21 & 15 & 108 \\
\hline Golpeteo & 23 & 15 & 15 & 24 & 21 & 14 & 112 \\
\hline Total & 55 & 38 & 40 & 55 & 48 & 35 & 271 \\
\hline
\end{tabular}

información dada por el personal del área de Manejo Integrado de Plagas y Enfermedades (MIPE) de cada finca, según el registro de las zonas que presentaban mayor incidencia de trips, además se tuvo en cuenta, que dichos puntos de muestreo, fueran áreas semi-naturales, con presencia de flora pratense y cercas vivas, representadas con especies de las familias Fabaceae, Poaceae y Rosaceae, en las cuales se ha reportado la presencia de especies del genero Orius (Falamarzi et al. 2009).

Para efectos de presentación de los resultados, según el municipio, a cada finca se le asignó un número: finca uno (Tocancipá), finca dos (Facatativá), finca tres (Cajicá), finca cuatro (Sopó), finca cinco (Chía) y finca seis (Sopo).

\section{Métodos de colecta de antocóridos}

Las colectas se realizaron entre los meses de marzo y julio del 2014, se evaluó cuatro métodos de colecta, esto con el propósito de determinar la eficiencia de los métodos de captura comúnmente empleados en entomología.

El primer método de colecta empleado fue el método de "golpeteo", el cual consintió en tomar las inflorescencias de la flora pratense y sacudirlas sobre una caja plástica, posteriormente los individuos fueron colectados por medio de un aspirador bucal, así como lo describe Bosco y Tavella (2008).

El segundo método, fue el usado por Guidone et al (2008), conocido como "Sacudida del follaje", para lo cual se realizó algunas modificaciones, que consistieron en realizar la sacudida del follaje de árboles y/o arbustos presentes en las cercas vivas, sobre el suelo, bajo el árbol y/o arbusto, se dispuso de una paño entomológico blanco, y los individuos que caían sobre este eran colectados por medio de un aspirador bucal.

El tercer método de colecta fue por "jameo", para el cual se usó una red entomológica de arrastre $(40 \mathrm{~cm} \varnothing$ y $90 \mathrm{~cm}$ de largo), con la cual se realizaron, tres veces, 40 pases dobles, en transectos lineales, en cada punto de muestreo en la que se empleó. El último método de muestreo consistió en trampas de caída tipo "pitfall", las cuales consistieron en frascos de vidrio de $15 \mathrm{~cm}$ de alto, con una boca de $5 \mathrm{~cm}$ de diámetro, los cuales se enterraron al nivel de la superficie del suelo. En el interior se colocó una solución de agua con detergente con 
el propósito de romper la tensión superficial, para evitar que los individuos capturados escaparan y también para retardar su descomposición (Culebra et al. 2009); las trampas permanecieron durante 24 horas por punto de muestreo.

No fue posible emplear el método de sacudida del follaje en todos los puntos de muestreo, porque no presentaban cercas vivas con árboles y/o arbustos. Por otro lado, el método de trampa de caída, solo se empleó en 30 puntos, cinco en cada una de las fincas floricultoras, en ninguno de estos puntos se colecto antocóridos. El número de veces que se empleó cada método de muestreo se muestra en la tabla 1.

Todos los individuos colectados fueron adultos y se guardaron vivos en frascos plásticos de $25 \mathrm{~mL}$ de volumen, debidamente rotulados y transportados al laboratorio de Control Biológico de la UMNG. Una vez en el laboratorio, 10 individuos de cada morfoespecie fueron preservados en alcohol al 70\%, para la posterior identificación taxonómica, los individuos restantes fueron empleados para establecer las colonias de cría.

\section{Identificación taxonómica de los antocóridos}

Los individuos preservados en alcohol se clasificaron por morfoespecie, por medio de observaciones de características morfológicas externas que son diagnosticas entre las especies de antocóridos, tales como coloración de los hemiélitros, caracteres diagnósticos de los artejos de las antenas y de las patas (Falamarzi et al. 2009).

Los individuos de cada una de las morfoespecies se sometieron a un proceso de aclaramiento, para lo cual se siguió la metodología propuesta por Jung et al. (2011) con algunas modificaciones, para lo cual, los individuos fueron sumergidos en una solución al $10 \%$ de $\mathrm{KOH}$ a temperatura ambiente durante tres días aproximadamente, hasta que los órganos del cuerpo se pudieran observar. Después se pasaron por agua destilada, en dos pases de un minuto cada uno. Posterior a esto, fueron colocados en ácido acético glacial durante 20 minutos.

Al finalizar el proceso de aclaramiento, los individuos se montaron en portaobjetos adicionándole solución Hoyers, tras lo cual la muestra fue sellada. Las muestras obtenidas fueron observadas bajo el microscopio. Se realizaron comparaciones de las características corporales más empleadas para la identificación de antocóridos, tales como la base del metasterno, características de los hemélitros, pronoto y los genitales de ambos sexos, de acuerdo con los resultados obtenidos por Ferragut y González (1994), Paterno et al. (2003); Shapiro et al. (2010) y Jung et al. (2011), los cuales realizaron identificaciones taxonómicas de diferentes especies de Orius spp. Además, se consultaron las claves propuestas por Froeschner (1949), Kelton (1978) y Pericat (1972).

Para la determinación final de la especie de los individuos colectados, se emplearon las características tanto del parámero del macho, como de la genitalita de la hembra, los cuales son los caracteres diagnósticos más empleados para la identificación de las especies de antocóridos, el parámero de los machos, es el carácter más distintivo y fácil de identificar según Yasunaga (1997), así como las características de la genitalia de las hembras, tales como la disposición del tubo copulador respecto a la línea media longitudinal del abdomen, caso en el que el tamaño y forma son un carácter diagnostico importante (Jung et al. 2011).

\section{Capacidad de depredación de}

los antocóridos colectados

\section{Cría de los depredadores}

Posterior a la identificación taxonómica de las especies de antocóridos colectadas, se establecieron colonias separadas para cada una de estas, los individuos fueron mantenidos en frascos de vidrio de $500 \mathrm{~cm}^{3}$, y alimentados con huevos de Sitotogra 
cerealella (Olivier) (Lepidoptera: Gelechiidae). Como sustrato de ovoposición se utilizaron vainas de Phaseolus vulgaris L, las colonias se mantuvieron en un cuarto de cría, dentro del laboratorio de Control Biológico de la UMNG en condiciones constantes $\left(26^{\circ} \mathrm{C} \pm 1{ }^{\circ} \mathrm{C}, 65 \pm 10 \%\right.$ HR y $\left.12 \mathrm{~L}: 12 \mathrm{D}\right)$,

\section{Determinación de la capacidad depredadora}

Con los individuos de cada una de las tres especies mantenidos en las colonias de cría, se realizó la evaluación de la capacidad depredadora. Para lo cual se emplearon como unidades experimentales cajas de Petri de $105 \mathrm{~mm}$ de diámetro, en la superficie interna de las cuales se colocó papel absorbente y sobre éste un circulo de hoja de frijol ( $P$. vulgaris $L$ ) de $5 \mathrm{~cm}$ de diámetro. Como presas se emplearon trips adultos de la especie F. occidentalis obtenidos del pie de cría mantenido en el laboratorio de Control Biológico de la UMNG, en cada unidad experimental se dispuso 40 trips y un adulto de antocórido (De dos a tres días de edad), el cual permaneció previamente en inanición durante un periodo de 12 horas.

Se contó con 10 repeticiones para cada una de las tres especies de antocórido evaluada, y un control positivo, el cual consistió en una unidad experimental con ausencia del depredador. Después de 24 horas de haber montado las unidades experimentales se realizó el conteo de los trips vivos y muertos.

Con los datos obtenidos se realizó un análisis de varianza (ANOVA). Con los residuales se evaluó el supuesto de normalidad, mediante la prueba de Shapiro-Wilk, y finalmente se realizó una comparación de medias, mediante la prueba de Tukey al 5\% de significancia $(p<0.05)$, para lo anterior se empleó el software de libre distribución R-project 2.15.2 @.

\section{RESULTADOS}

En este estudio, se colectaron tres especies de antocóridos, los cuales se clasificaron en tres morfoespecies basados en el tamaño corporal, la coloración de los hemélitros y características de los artejos antenales, para determinar la subfamilia a la que pertenecía cada una de estas morfoespecies, se empleó la clave taxonómica propuesta por Kelton (1978).

Dos de las morfoespecies se agruparon dentro de la familia Anthocorinae (Reuter), mientras que la otra fue agrupada en la subfamilia Lyctororinae (Reuter), lo anterior debido a que el tercer y cuarto segmento de las antenas eran filiformes y presentaban pelos más largos que el doble del diámetro de los segmentos, en contraste, los individuos de la subfamilia Anthocorinae, presentaron pelos en el tercer y cuatro segmentos de las antenas, más cortos que el doble del diámetro de los segmentos y estos fueron fusiformes.

La especie perteneciente a la familia Lyctocorinae no se identificó a nivel de especie, porque el número de individuos colectados fue muy bajo (cuatro individuos), los cuales se emplearon exclusivamente para establecer la colonia de cría, a diferencia de las otras dos especies, que al haber capturado mayor número de individuos, se pudo disponer de algunos ejemplares para sacrificarlos y procesarlos para hacer la respectiva identificación taxonómica.

Las dos especies de la subfamilia Anthocorinae, presentaron cuello corto y base de la cabeza no prolongada, por lo que se clasificaron dentro del género Orius (Figura 1A-B y figura 2A-B); las especies del genero Orius están agrupadas dentro de un grupo muy homogéneo morfológicamente (Ferragut y Gonzales, 1994), por esto, como carácter diagnóstico para su identificación a nivel de especie se tuvo en cuenta las características del parámero de los machos y la genitalia de las hembras.

Una de las especies del genero Orius, fue identificada como Orius insidiosus (Say), la cual se caracterizó por presentar un parámero con cono hinchado en la región proximal y cónico en la región distal, flagelo corto y en forma de hoja casi tan largo 
y ancho como el cono (Figura 1D), la genitalia de la hembra presento tubo copulador cilíndrico, con igual ancho que largo y con disposición casi paralela respecto a la línea longitudinal media del abdomen (Figura 1E) (Shapiro et al. 2010).

La otra especie se identificó como Orius pumilio (Champion), a diferencia de $O$. insidiosus, el parámero presento tanto un cono y flagelo más largo, este último se caracterizó por ser filiforme en la región distal y por presentar una espina en la región medial (Figura 2D), la genitalia de la hembra presento un tubo copulador aproximadamente del doble de longitud que el ancho, y su disposición respecto a la línea longitudinal media del abdomen fue inclinada hacia el lado opuesto (Figura 2E) (Shapiro et al. 2010).
En los muestreos realizados, sin discriminar el método de colecta empleado, en total se colectaron 394 individuos, la especie 0 . insidiosus fue la más abundante, en la que se agrupó el 75\% de los individuos colectados, seguidos por la especie $O$. pumilio y la especie de la subfamilia Lyctocorinae en la que se agruparon el $24 \%$ y el $1 \%$ del total de los individuos colectados respectivamente.

Entre las seis fincas floricultoras, en total fueron muestreados 113 puntos, en 54\% de estos, no se colectó ningún individuo, mientras que en el 19\% se colectaron individuos tanto de la especie 0 . insidiosus como de la especie O. pumilio, en el $18 \%$, $7 \%$ y el $2 \%$ de los muestreos se colectaron únicamente individuos de las especies $O$. insidiosus, $O$. pumilio, e individuos de la subfamilia Lyctocorinae, respectivamente.
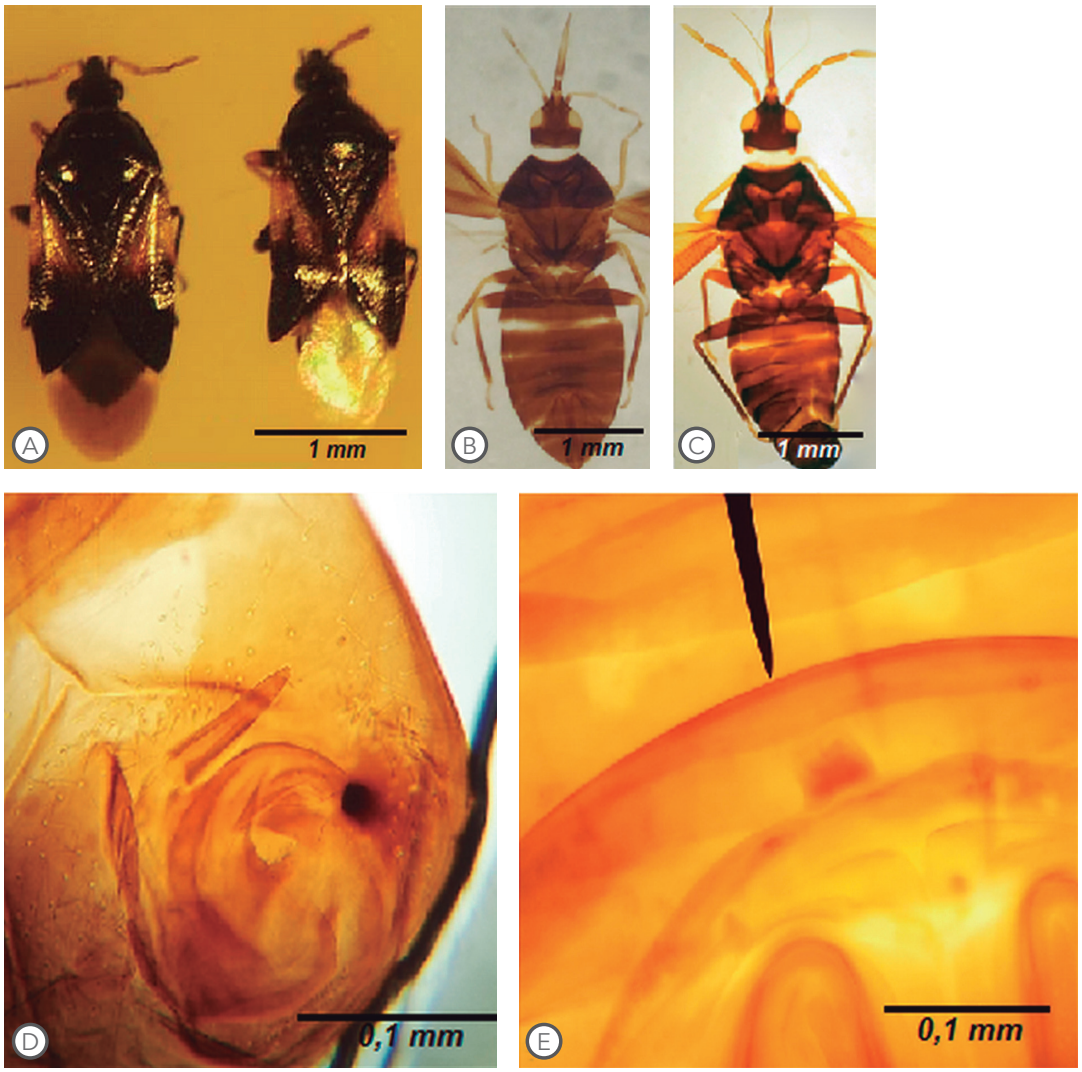

Figura 1. Orius insidiosus. A: Adultos, Izq-Hembra, Der-Macho; B. Hembra después del proceso de aclaramiento: C: Macho después del proceso de aclaramiento: D: Parámero del macho: E: Genitalia de la hembra. 

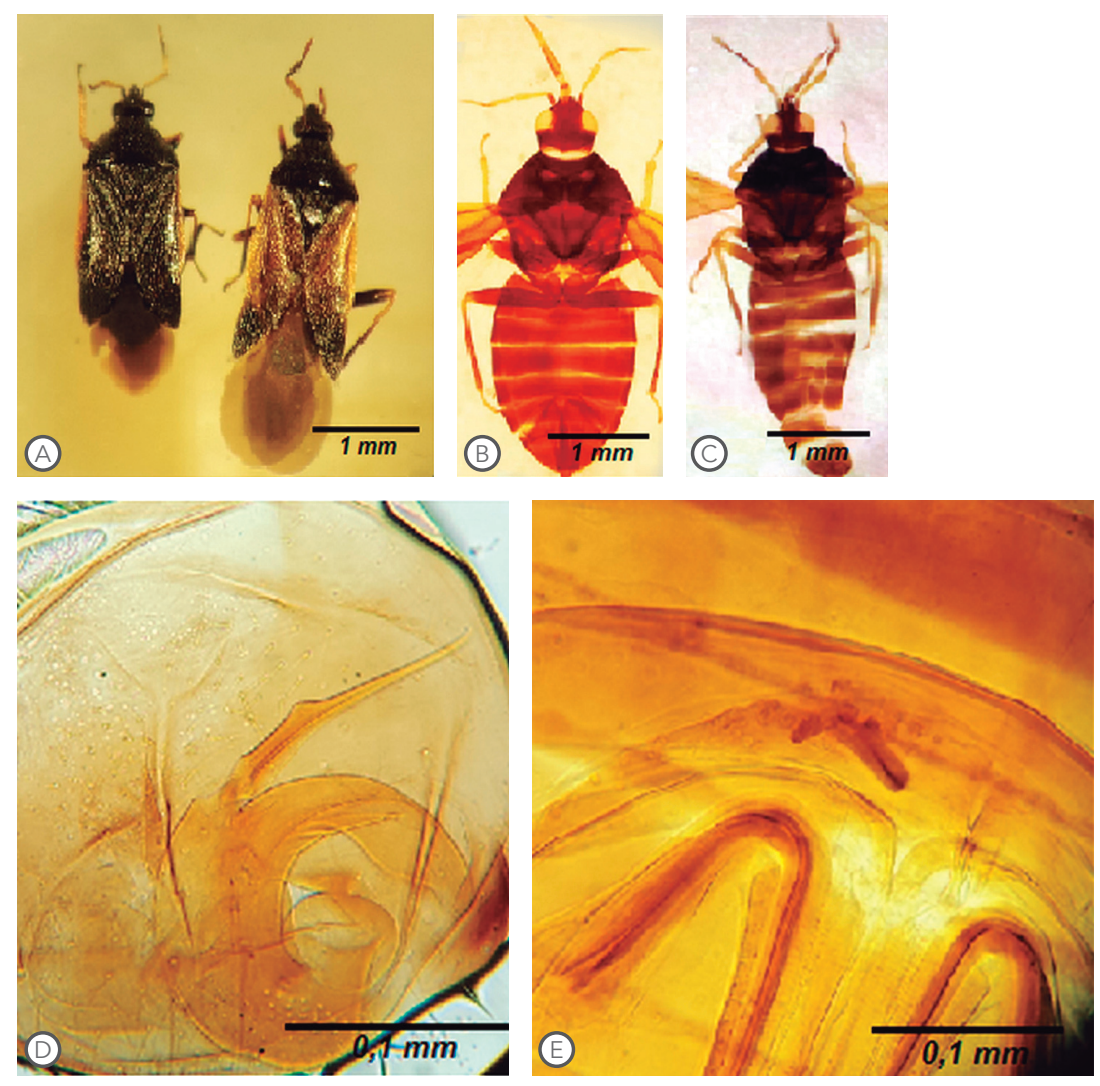

Figura 2. Orius pumilio. A: Adultos, Izq-Hembra, Der-Macho; B. Hembra después del proceso de aclaramiento: C: Macho después del proceso de aclaramiento: D: Parámero del macho: E: Genitalia de la hembra.

En todas las seis fincas en donde se realizaron muestreos hubo presencia tanto de $O$. insidiosus, como de O. pumilio, mientras que solo en las fincas uno y seis, hubo presencia de la especie de la subfamilia Lyctocorinae, los individuos de esta subfamilia fueron encontrados exclusivamente en puntos de muestreo próximos a pilas de compost. En la finca cinco, se encontró el mayor número promedio de individuos de antocóridos, el cual fue diferente significativamente al número promedio de individuos de antocóridos encontrados en las cinco fincas restantes (Figura 3).

En la figura 4, se puede observar que el número promedio de individuos colectados de la especie O. insidiosus fue significativamente mayor en la finca número 5, mientras que para la especie 0 . pumilio no se presentaron diferencias significativas en el número promedio de individuos colectados en las seis fincas floricultoras.

El método de muestreo con el que se colectó mayor número de antocóridos fue el de golpeteo, con el que se obtuvo más de la mitad de las capturas. Con el método de jameo se logró colectar el $33 \%$ de los individuos, mientras que con los métodos de sacudida del follaje y trampa de caída no se colectaron individuos (Figura 5).

Como se observa en la Figura 6, la especie O. insidiosus presentó una capacidad de depredación media de $23 \pm 5,29$ trips adultos de F. occidentalis en un periodo de 24 horas, lo cual fue 
significativamente mayor $(p<0,05)$, respecto a la capacidad de depredación registrada tanto para la especie de la subfamilia Lyctocorinae como para la especie $O$. pumilio, las cuales presentaron un consumo medio de 10,5 5,32 y 6,2 $\pm 6,55$ trips $F$. occidentalis en 24 horas respectivamente. Por otro lado, en el tratamiento control se registró una mortalidad de 1,11 $\pm 1,54$ trips $F$. occidentalis, lo cual no fue diferente significativamente respecto al tratamiento en el que se empleó la especie $O$. pumilio.

\section{DISCUSIÓN}

En la sabana de Bogotá, los trabajos reportados sobre el muestreo y colecta de especies de la familia Anthocoridae se reducen a los estudios realizados por Zenner (2011), quien reportó la presencia de adultos y ninfas de Orius spp. en especies de plantas con flores, el trabajo realizado por Chacón (2002), quien identificó tres especies de antocóridos colectados el municipio de Chía, y el trabajo realizado por Osorio (2001), quien colectó e identifico una morfoespecie de la familia Anthocoridae en el municipio de Mosquera.

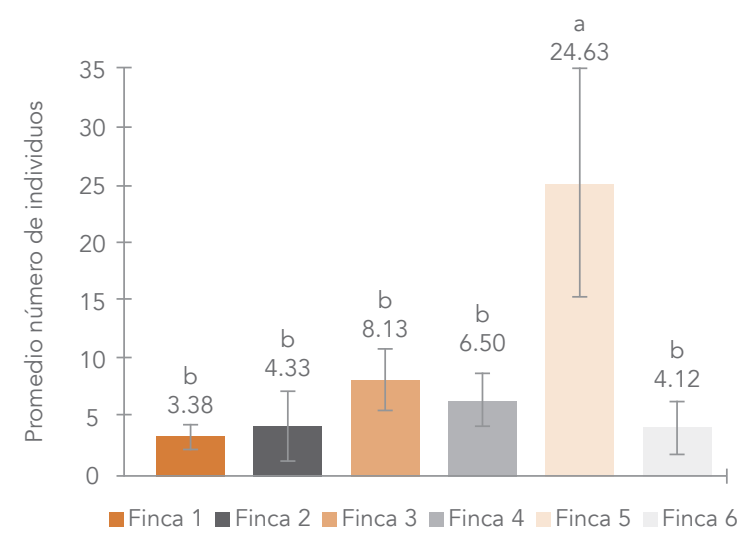

Figura 3. Número medio de antocóridos colectados en cada una de las seis fincas ( \pm Error estándar).

Columnas con diferente letra indican diferencias significativas (PruebaTukey $p<0.05$ )

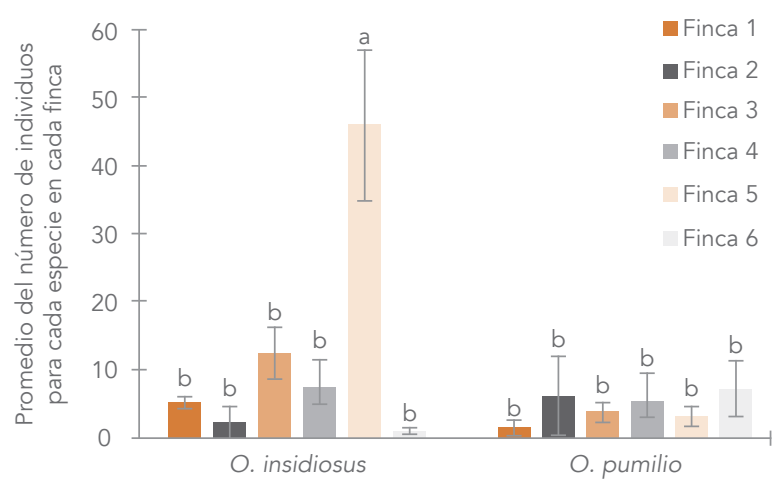

Figura 4. Número medio de individuos colectados en cada una de las fincas, para las dos especies del genero Orius ( \pm Error estándar). Columnas con diferente letra indican diferencias significativas (Prueba Tukey $p<0.05)$.

Al comparar los resultados mencionados anteriormente, con los resultados del número de especies colectadas e identificadas en este estudio, se podría pensar que el número de especies de la familia Anthocoridae encontradas en fincas productoras de flores de la sabana de Bogotá es bajo.

Sin embargo, es de resaltar que la incidencia natural de estos depredadores en las áreas seminaturales ubicadas en las fincas floricultoras, es importante, porque indica la adaptación de estas especies en tales áreas, pese a las condiciones adversas de dichos agroecosistemas, generadas por la alta presión química, aunque también existen algunas condiciones ambientales favorables en ellos, pues las plantas hospederas presentes en las áreas semi-naturales, se constituyen en un hábitat refugio para los artrópodos, al ofrecer entre otras cosas, alimento, sitios de apareamiento, y protección ante insecticidas químicos (Bosco y Tavela, 2013) .

La presencia de los antocóridos en las fincas floricultoras, puede tener un efecto positivo, frente a la regulación de las poblaciones de trips que migran desde las áreas circundantes hacia dentro de los invernaderos. Se ha demostrado el efecto 
- Sacudida del follaje Jameo Golpeteo-Trampa de caida

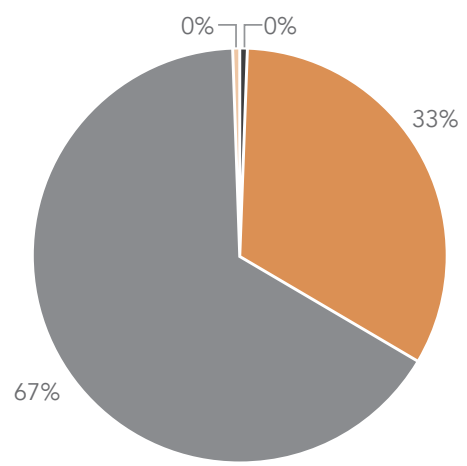

Figura 5. Porcentaje del número de individuos colectados con cada uno de los métodos utilizados en el estudio.

positivo de las áreas semi-naturales en la diversidad y/o abundancia de enemigos naturales de plagas (Veres et al. 2013).

Al tener en cuenta que en el 19\% de los 113 puntos muestreados se colectaron individuos, tanto de la especie $O$. insidiosus como $O$. pumilio y con base a lo reportado por Shapiro et al. (2009), se puede sugerir que estas dos especies pueden llegar a tener una coexistencia temporal en simpatría. No obstante, al tener en cuenta que el número de individuos colectados de la especie $O$. insidiosus fue aproximadamente tres veces mayor, respecto al número de individuos colectados de la especie O. pumilio, y que el número promedio de individuos colectados en una de las fincas fue significativamente mayor para el caso de 0 . insidiosus (Figura 4), se podría pensar que la competencia por el recurso entre estas dos especies lleva a que se presente dominancia por O. insidiosus.

Por otro lado la tercera especie identificada, perteneciente a la subfamilia Lyctocorinae, solo fue encontrada en pilas de compost en dos de las seis fincas, esto no había sido reportado hasta el momento y es importante porque generalmente en las pilas de compost no se hace ningún tipo de control para los trips presentes, por lo tanto este depredador puede ejercer un papel importante en el control de las poblaciones de la plaga ubicadas allí.

Pese a lo anterior, hay que tener en cuenta que esta especie seguramente tiene otra fuente de alimento diferente a trips, debido a que en el ensayo de capacidad de depredación se observó que consume bajo número de trips respecto a $O$. insidiosus (Figura 6), por tal motivo y sumado a que no se identificó hasta el nivel taxonómico de especie, se hace necesario realizar dicha identificación, con el propósito de analizar el rol que cumple o puede cumplir en las pilas de compost, el cual puede estar relacionado o no con el control de trips.

Como una aproximación a la posible especie a la que pertenece este antocórido, basados en las características morfológicas externas observadas en este estudio, sumado a reportes realizados en la región de Boyacá (Osorio 2001) y según el hábitat donde fue encontrada, esta especie puede corresponder, a la especie Lyctocoris campestris (Fabricius) (Osorio, 2001), la cual es una especie que se ha demostrado que tiene potencial como agente de control biológico para diferentes plagas (Bellows y Fisher, 1999).

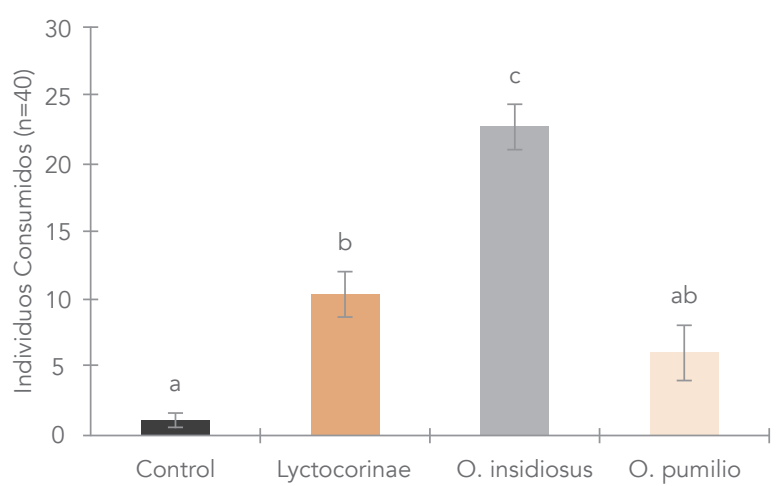

Figura 6. Número de trips $F$. occidentalis consumidos por un adulto de la especie de antocórido en 24 horas y nmero de trips muertos en el tratamiento control (Media \pm Error estandar, $n=40$ trips). Columnas con diferente letra indican diferencias significativas (Prueba Tukey $p<0.05$ ). 
En relación al número promedio de antocóridos encontrados en las seis fincas floricultoras, en la finca cinco ( $4^{\circ} 50^{\prime} 36^{\prime \prime} \mathrm{N}$ y $\left.74^{\circ} 03^{\prime} 22^{\prime \prime} \mathrm{W}\right)$, se encontró mayor número de antocóridos respecto a las demás fincas (Figura 3). Esto puede deberse a que la finca cinco, además de ser una finca floricultora, está dedicada también a la ganadería, por lo que tiene mayor presencia de áreas con pastizales y flora pratense, lo cual garantiza mayor área de hábitat donde pueden estar presentes tanto los antocóridos como los trips.

La presencia de trips, en estas áreas de pastizales cercanas a los cultivos de flores, puede deberse a que $F$. occidentalis se dispersa hacia los a cultivos y nuevas áreas, a partir de zonas próximas, con presencia de diferentes especies de plantas silvestres, que les suministran abundante recurso y donde tienden a encontrarse en cantidades importantes, (Groves et al. 2001).

Una de las especies silvestres, sobre la cual se encontró mayor presencia de antocóridos en este estudio fue el trébol rojo Trifolium pratense (Fabales: Fabaceae). En la mayoría de los muestreos sobre estas plantas, cuando se encontraron antocóridos, también se observó presencia de trips, esto se puede deber a que muchos depredadores polífagos presentan en muchos casos preferencia por consumir una presa en particular, cuando tienen disponibilidad de más de una presa al mismo tiempo (Xu y Enkegaard, 2009) .

En todas las fincas se encontró presencia de antocóridos de las dos especies del genero Orius (Figura 4), aunque no en todos los puntos de muestreo dentro de las fincas hubo presencia de estos. En muchos de los puntos de muestreo sin presencia de antocóridos se observó poca o nula presencia de trips, por lo tanto la presencia de estos depredadores puede estar condicionada a la presencia de la presa.

En este sentido, aunque los antocóridos son considerados depredadores, zoofitófagos generalistas
(Funderburk et al. 2000; Seagraves y Lundgren, 2010) se ha demostrado que estos depredadores pueden llegar a presentar preferencia por ciertas presas. Para el caso de las especies del genero Orius se ha demostrado preferencia por consumir F. occidentalis como presa. Reitz et al. (2006) menciona que los antocóridos del genero Orius se alimentan selectivamente de este fitófago, debido a esto, la ausencia de antocóridos en muchos de los puntos muestreados pueden deberse a la ausencia de trips.

El análisis realizado para la determinación del método de muestreo más eficiente, en términos de número de capturas, puede resultar de gran valor para el diseño de metodologías para la colecta de antocóridos a futuro, ya sea en fincas floricultoras o en áreas aledañas a estas. Respecto a los resultados aquí obtenidos (Figura 5), no se colecto ningún individuo empleando los métodos de trampa de caída y sacudida del follaje, esto pudo deberse a respuestas de características ecológicas o etológicas que presentan los diferentes grupos de insectos, lo que condiciona su presencia a diferentes microhabitats (Marrero et al. 2008).

Sumado a lo anterior, y al saber que gran mayoría de las especies de antocóridos, habitan sobre el follaje y ramas de arbustos y árboles (Kelton, 1978), pero principalmente sobre las cabezas de floración de especies herbáceas o arbustivas (Tommasini, 2004), el comportamiento ecológico de los antocóridos, puede sustentar los resultados encontrados en este trabajo, debido a que los valores más altos de eficiencia y proporción de individuos colectados, se obtuvieron para el método de golpeteo (Figura 5), el cual se realizó exclusivamente sobre flores de plantas herbáceas y arbustivas, y el método de jameo, con el cual se realizó arrastres superficiales sobre las flores de especies herbáceas.

La especie $O$. insidiosus presento la mayor capacidad de depredación respecto las demás 
especies, la mortalidad generada por el consumo de la especie $O$. pumilio, no mostro efecto diferente a la mortalidad natural de los trips observada en el tratamiento control, con lo cual se podría pensar, que el potencial depredador y de eficiencia de búsqueda de sus presa es bajo. Sin embargo este resultado debe ser corroborado mediante experimentación adicional, pues según a lo reportado por Tommasini et al. (2004), el potencial de consumo de una especie se determina mediante la evaluación de la tasa de consumo de la especie depredadora, en diferentes densidades de la presa, es decir ensayos de respuesta funcional.

Por este motivo, en conclusión, los resultados de la capacidad de depredación de los antocóridos sobre los adultos de trips F. occidentalis, son una buena base para realizar ensayos de respuesta funcional. Por otro lado la presencia e incidencia natural de las especies de antocóridos encontradas en el presente estudio, indica que estas poblaciones se han mantenido dentro de estos agroecositemas, ejerciendo una presión sobre las poblaciones de trips.

\section{AGRADECIMIENTOS}

Agradecemos al personal de las fincas floricultoras que nos acompañaron en las colectas del material biológico durante la investigación y a las entidades financiadoras. "Producto derivado del proyecto CIAS 1654, financiado por la Gobernación de Cundinamarca, la Asociación Colombiana para el Avance de la Ciencia bajo el contrato de financiamiento 097 del 2013 y cofinanciado por la Vicerrectoría de investigaciones de la Universidad Militar Nueva Granada".

\section{REFERENCIAS}

1. Allen W R y Broadbent B. 1986. Transmission of tomato spotted wilt virus in Ontario greenhouses by Frankliniella occidentalis. Canadian Journal of Plant Pathology, 8: 33-38.

2. Bajonero J, Córdoba N, Cantor F, Rodríguez D, Cure J R 2008. Biología y ciclo reproductivo de Apanteles gelechiidivoris (Hymenoptera: Braconidae), parasitoide de Tuta absoluta (Lepidoptera: Gelechiidae). Agronomia Colombiana, 26(3): 417- 426.

3. Bellows T S, Fisher T W. 1999. Handbook of Biological Control: Principles and Applications. Academic Press, San Diego, 1049 p.

4. Bielza P. 2008. Insecticide resistance management strategies against the western flower thrips, Frankliniella occidentalis. Pest Management Science, 64: 1131-1138

5. Bosco L, Giacometto E, Tavella L. 2007. Colonization and predation of thrips (Thysanoptera: Thripidae) By Orius spp. (Heteroptera: Anthocoridae) in sweet pepper greenhouses in Northwest Italy. Biological control, 44: 331-340.

6. Bosco L, Tavella L. 2008. Collection of Orius species in horticultural areas of northwestern Italy. Bulletin of Insectology, 61 (1): 209-210.

7. Bosco L, Tavella L. 2013. Distribution and abundance of species of the genus Orius in horticultural ecosystems of northwestern Italy. Bulletin of Insectology, 66 (2): 297-307.

8. Calixto A C. 2005. Trips del suborden Terebrantia (Insecta: Thysanoptera) en la sabana de bogotá. Revista Colombiana de Entomología, 31(2): 207-213.

9. Castresana J, Gagliano E, Puhl L, Bado S, Vianna L y Castresana M. 2008. Atracción del trips Frankliniella occidentalis (Pergande) (Thysanoptera: Thripidae) con trampas de luz en el cultivo de Gerbera jamessonii (G.). Idesia, 26(3): 51-56. 
10. Chacón E D. 2002. Búsqueda de depredadores nativos de trips en la sabana de Bogotá y evaluación de su eficacia sobre Frankliniella occidentalis (Pergande) (Thysanoptera: Thiripidae) bajo condiciones de laboratorio. Trabajo de grado. Departamento de Biología, Facultad de ciencias. Universidad Nacional de Colombia. Bogotá.

11. Childers C, Achor D. 1995. Feeding and oviposition injuries to plants to economic plants, subsequent damage and host responses to infestation. p. 31-51. En: Lewis T (Ed.). Cab International, Burlington, $236 \mathrm{p}$.

12. Childers C C, Nakahara S. 2006. Thysanoptera (thrips) within citrus orchards in Florida: species distribution, relative and seasonal abundance within trees, and species on vines and ground cover plants. Journal of Insect Science, 6 1-19.

13. Culebra S, Catalano P, Sgarbi C, Verzero F, Blondel D, Ricci M, Antonini A. 2009. Utilización de trampas Pitfall con distintos atrayentes alimentarios para el monitoreo de hormiga en sistemas pastoriles. Boletín de sanidad vegetal - Plagas, 35(2): 187-192.

14. Demirozer O, Tyler-Julian K, Funderburk J, Lepplac N, Reitz S. 2012. Frankliniella occidentalis (Pergande) integrated pest management programs for fruiting vegetables in Florida. Pest Management Science. 68: 1537-1545

15. Falamarzi S, Asadi G, Hosseini R. 2009. Species inventory, preys and host plants of Anthocoridae sensu lato (Hemiptera: Heteroptera) in Shiraz and its environs (Iran, Fars province. Acta Entomologica musei Nationalis Pragae, 49(1): 33-42.

16. Ferragut F, González J E. 1994. Diagnóstico y distribución de las especies de Orius Wolff 1811, peninsulares (Heteroptera, Anthocoridae). Boletín de Sanidad Vegetal Plagas, 20: 89-101.

17. Froeschner R. 1949. Contribution to a sinopsis of the Hemiptera of Missouri, Pt. IV Hebridae,
Mesoveliidae, Cimicidae, Anthocoridae, Cryptostemmatidae, Isometopideae, Meridae. American Midland Naturalist 42(1): 123-188.

18. Funderburk J, Stavisky J, Olsen S. 2000. Predation of Frankliniella occidentalis (Thysanoptera: Thripidae) in field peppers by Orius insidiosus (Hemiptera: Anthocoridae). Environmental Entomology, 29: 376-382.

19. Groves R L, Walgenbach J F, Moyer J W, Kennedy G G. 2001. The role of weed hosts and tobacco thrips, Frankliniella fusca (Thysanoptera: Thripidae), in the epidemiology of tomato spotted wilt virus. Plant Disease. 86: 573-582.

20. Guidone L, Loru L, Marras P, Fois X, Pantaleoni R, Tavella L. 2008. Predatory bugs in hazelnut orchards of Piedmont and Sardinia (Italy). Bulletin of Insectology, 2008. 61 (1): 207-208.

21. Hill, D.S., 2008. Pests of Crops in Warmer Climates and Their Control. Springer Science \& Business Media, Skegness, $704 \mathrm{p}$.

22. Hunter W B, Ullman D E. 1989. Analysis of mouthpart movements during feeding of Frankliniella occidentalis (pergande) and F. schultzei trybom (Thysanoptera: Thripidae). International Journal of Insect Morphology and Embryology, 18 (2-3): 161-171.

23. Johansen R, Mojica A. 2007. About some thrips species assemblages found in avocado trees (Persea americana Mill) in México. VI World Avocado Congress. Viña Del Mar, Chile.

24. Jung S, Yasunaga T, Lee S. 2011. Taxonomic review of the genus Orius (Heteroptera: Anthocoridae) in the Korean Peninsula. Journal of Asia-Pacific Entomology, 14: 64-74.

25. Kelton L A. 1978. The insect and arachnids of Canada. Part 4, the Anthocoridae of Canada and Alaska. Kromar Printing Ltd, Ottawa, $101 \mathrm{p}$.

26. Marrero H J, Zalba S M, Carpintero D L. 2008. Eficiencia relativa de distintas técnicas de captura de heterópteros terrestres en un pastizal de montaña. BioScriba. 1(1): 3-9. 
27. Lewis T. 1997. Thrips as crop pests. Firts Edition. Cab International, Wallingford, 736 p.

28. Mendoza P. 2014. Interceptaciones por Trips. I Simposio: Control Biológico de Trips. Mosquera, 30-40 p.

29. Osorio A. 2001. Reconocimiento de enemigos naturales de Tecia solanivora (Povolny) (Lepidoptera: Gelechiidae) en localidades productoras de papa en Colombia. Revista Colombiana de entomología, 27: 177-185.

30. Parker B L, Skinner M, Lewis T. 1995. Thrips biology and management. Plenum Press, Burlington, $276 \mathrm{p}$.

31. Paterno L C, Paes V H, Martins S. 2003. Record of two species of Orius Wolff (Hemiptera, Anthocoridae) in Brazil. Revista Brasileira de Entomología, 47(2): 303-306.

32. Pericart J. 1972. Hémiptères. Anthocoridae, Cimicidae et Microphsidae de l'ouest-paléarctique.- Masson, Paris.

33. Reitz S R. 2009. Biology and Ecology of the Western Flower Thrips (Thysanoptera: Thripidae): The Making of a Pest. Florida Entomologist, 92(1): 7-13.

34. Seagraves M, Lundgren J. 2010. Oviposicion response by Orius insidiosus (Hemiptera: Anthocoridae) to plant quality and prey availability. Biological control, 55:174-177.

35. Shapiro J P, Shirk P D, Kelley K, Lewis T M, Horton D. R. 2010. Identity of two sympatric species of Orius (Hemiptera: Heteroptera: Anthocoridae). Journal of Insect Science, 10: 1-17.

36. Shapiro J, Shirk P, Reitz S, Koenig R. 2009. Sympatry of Orius insidiosus and O.pumilio (Hemiptera: Anthocoridae) in North Central Florida. Florida Entomologist, 92 (2):362-366.

37. Shipp J, Ramakers P. 2004. Biological control of thrips on vegetable crops. In: Heinz K, Van Driesche R., AndParrella M. Editors, Biocontrol in Protected Culture. Ball Publishing. Batavia. 265-276 pp.
38. Tenjo, F., Montes, E., Martínez, J. 2006. Comportamiento reciente (2000-2005) del sector floricultor Colombiano. Banco de la Republica, Bogotá, 22 p.

39. Tommasini MG, Maini S. 1995. Frankliniella occidentalis and other thrips harmful to vegetable and ornamental crops in Europe. Agricultural University Wageningen Papers, 95: $1-42$.

40. Van Lenteren J, Roskam M, Timmer R. 1997. Commercial Mass Production and Pricing of Organisms for Biological Control of Pests in Europe. Biological Control, 10 (2): 143-149.

41. Veres A, Petit S, Conord C, Lavigne C, 2013. Does landscape composition affect pest abundance and their control by natural enemies? A review. Agriculture, Ecosystems \& Environment, 166:110-117.

42. Vergara R R. 1998. El Thrips palmi Karny nueva plaga de la agricultura Colombiana. Comité Departamental de Thrips palmi, Medellín, $163 \mathrm{p}$.

43. Xu X, Enkegaard A. 2009. Prey preference of Orius sauteri between Western Flower Thrips and spider mites. Entomologia Experimentalis et Applicata, 132 (1):93-98.

44. Yasunaga T. 1997. The flower bug Genus Orius Wolff (Heteroptera: Anthocoridae) from Japan and Taiwan, part I. Applied Entomological Zoology, 32 (2): 355-364.

45. Zapata AM, Hincapie Y, Madrigal A. 1994. Reconocimiento de Thrips en cultivos de flores y áreas aledañas y biología de Frankliniella panamensis Hood (Thysanopiera: Thripidae). Revista Colombiana de Entomologia, 20(1): 47-52.

46. Zenner I. 2011. Universidad de ciencias aplicadas y ambientales U.D.C.A Bogotá. D.C. Facultad de ingeniería agronómica. Notas y Noticias Entomológicas, 31(1): 1-19. 